\title{
A Semi-Analytical Method for the Solution of Helmholtz Equation
}

\author{
M. Tadi \\ Department of Mechanical Engineering, University of Colorado at Denver, Denver, USA \\ Email:mohsen.tadi@ucdenver.edu
}

Received October 2, 2013; revised October 30, 2013; accepted November 5, 2013

Copyright (C) 2013 M. Tadi. This is an open access article distributed under the Creative Commons Attribution License, which permits unrestricted use, distribution, and reproduction in any medium, provided the original work is properly cited.

\begin{abstract}
This note is concerned with a semi-analytical method for the solution of 2-D Helmholtz equation in unit square. The method uses orthogonal functions to project the problem down to finite dimensional space. After the projection, the problem simplifies to that of obtaining solutions for second order constant coefficient differential equations which can be done analytically. Numerical results indicate that the method is particularly useful for very high wave numbers.
\end{abstract}

Keywords: Helmholtz Equation; Elliptic Systems; High Wavenumber

\section{Introduction}

In this note we consider a numerical method for the solution of 2-D Helmholtz equation. The goal is provide a solution method that is more suitable for Helmholtz equation at high frequencies and can be applied to the Helmholtz equation in 3-D.

Helmholtz equation appears very naturally in the study of wave propagation [1], after assuming a harmonic field. It is well-known that the numerical solution of the Helmholtz equation is a challenging problem for high frequencies. Higher order finite difference schemes have been developed for Helmholtz equation with homogeneous domain [2-4]. Additional methods such as boundary element [5], discontinuous Galerkin method [6], multilevel multi-integral algorithm [7], iterative methods [8], and methods based on parallel computing [9] have also been developed for the numerical solution of Helmholtz equation. Recent results also include an iterative method based on ADI [10], a finite-element based semi-analytic method [11], and a method based on discrete singular convolution [12].

The method presented in this note is based on orthogonal functions. In addition to good accuracy for high wave numbers, it has the following specific new features.

- The actual calculation is performed analytically in 1-D only.

- It can also be applied to 3-D domains.

Section 2 introduces the basic principles of the method.
Section 3 presents the numerical results and Section 4 is devoted to the concluding remarks.

\section{Mathematical Formulations}

Consider a 2-D Helmholtz equation given by

$$
\begin{gathered}
u_{x x}+u_{y y}+k^{2} u=0, x, y \in[0,1], \\
u(0, y)=f_{w}(y), u(1, y)=f_{e}(y), \\
u(x, 0)=f_{s}(x), u(x, 1)=f_{n}(x),
\end{gathered}
$$

where, for simplicity, the domain is assumed to be a unit square. A set of Dirichlet boundary conditions are also given. In the present method, the solution to the above system is obtained as a linear combination of two similar problems. This is only to satisfy nonzero boundary conditions (Figure 1).

The two problems can now be similarly treated. Consider the first problem, where the boundary conditions at $x=0$ and $x=1$ are set equal to zero. Consider an expansion of the solution in the form

$$
u(x, y)=\sum_{j=1}^{\infty} f_{i}(y) \sin (j \pi x)
$$

where, the zero boundary conditions are satisfied automatically. A similar formulation can be used for the Helmholtz equation in 3-D (Appendix). Multiplying both sides of Equation (2) by $\sin (j \pi x)$, integrating both sides fover the domain $[0: 1]$, and using the orthogonal- 


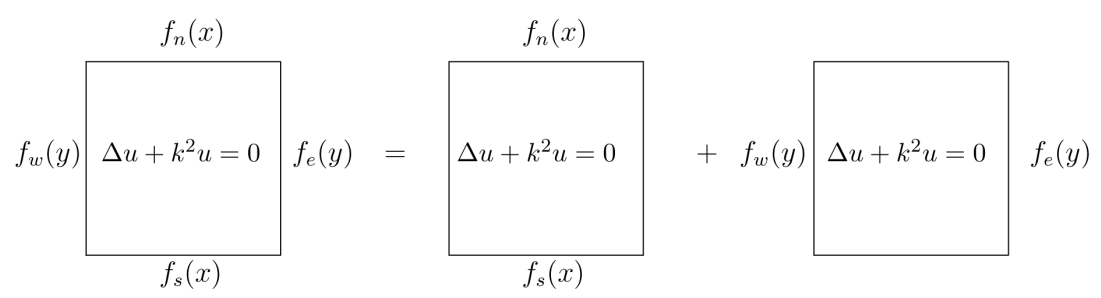

Figure 1. The decomposition of the elliptic problem to two elliptic problems with apporipriate boundary conditions.

ity condition

$$
\int_{0}^{1} \sin (i \pi x) \sin (j \pi x) d x= \begin{cases}\frac{1}{2} & \text { if } i=j \\ 0 & \text { otherwise }\end{cases}
$$

it is possible to obtain a relationship for the functions $f_{j}(y)$ given by

$$
f_{j}(y)=2 \int_{0}^{1} u(x, y) \sin (j \pi x) \mathrm{d} x .
$$

Differentiating the above equation with respect to $y$ twice and using Equation (1) leads to

$$
\frac{\mathrm{d}^{2}}{\mathrm{~d} y^{2}} f_{j}(y)=-2 \int_{0}^{1} u_{x x} \sin (j \pi x) \mathrm{d} x-2 k^{2} \int_{0}^{1} u \sin (j \pi x) \mathrm{d} x .
$$

Integrating the first integral on the right-hand side by parts twice leads to

$$
\begin{aligned}
\frac{\mathrm{d}^{2} f_{j}}{\mathrm{~d} y^{2}}= & -2\left[\left.u_{x} \sin (j \pi x)\right|_{0} ^{1}+(j \pi)\left[\left.u \cos (j \pi x)\right|_{0} ^{1}\right.\right. \\
& \left.\left.+(j \pi) \int_{0}^{1} u \sin (j \pi x) \mathrm{d} x\right]\right]-2 k^{2} \int_{0}^{1} u \sin (j \pi x) \mathrm{d} x .
\end{aligned}
$$

After applying the boundary conditions, the above equation simplifies to

$$
\frac{\mathrm{d}^{2}}{\mathrm{~d} y^{2}} f_{j}(y)+\left(k^{2}-(j \pi)^{2}\right) f_{j}(y)=0 .
$$

The above equation is now a simple constant coefficient second-order differential equation. The appropriate boundary conditions are given by

$$
\begin{aligned}
& f_{j}(0)=2 \int_{0}^{1} u(x, 0) \sin (j \pi x) \mathrm{d} x, \\
& f_{j}(1)=2 \int_{0}^{1} u(x, 1) \sin (j \pi x) \mathrm{d} x .
\end{aligned}
$$

The second-order differential equation can be solved. There are three separate cases.

Case 1: $\left(k^{2}>(j \pi)^{2}\right)$, in this case the solution is given by

$$
f_{j}(y)=f_{j}(0) \cos (\sigma y)+\frac{f_{j}(1)-f_{j}(0) \cos (\sigma)}{\sin (\sigma)} \sin (\sigma y),
$$

where, $\sigma=\sqrt{k^{2}-(j \pi)^{2}}$
Case 2: $\left(k^{2}<(j \pi)^{2}\right)$, in this case the solution is given in terms of exponential function. One needs to consider two separate cases.

- $\left((j \pi)^{2}-k^{2}\right)=$ small, or $\mathrm{e}^{\left((j \pi)^{2}-k^{2}\right)} \ll \infty$. In this case the solution is given by

$$
f_{j}(y)=\frac{f_{j}(1)-\mathrm{e}^{-\sigma} f_{j}(0)}{\mathrm{e}^{\sigma}-\mathrm{e}^{-\sigma}} \mathrm{e}^{\sigma y}+\frac{f_{j}(1)-\mathrm{e}^{\sigma} f_{j}(0)}{\mathrm{e}^{-\sigma}-\mathrm{e}^{\sigma}} \mathrm{e}^{-\sigma y},
$$

where, $\sigma=\sqrt{\left((j \pi)^{2}-k^{2}\right)}$.

$\left((j \pi)^{2} \gg k^{2}\right)$ For this case, in order to avoid unbounded functions, one needs to approximate $\mathrm{e}^{-\sigma} \approx 0$. For this case, the solution is given by

$$
f_{j}(y)=f_{j}(1) \mathrm{e}^{-\sigma(1-y)}+\left(f_{j}(0)-f_{j}(1) \mathrm{e}^{-\sigma}\right) \mathrm{e}^{-\sigma y} .
$$

Case 3: $\left(k^{2} \approx(j \pi)^{2}\right)$ This is the singular case. For these cases, it is possible to treat the differential equation according to the following. For $\epsilon=\left(k^{2}-(j \pi)^{2}\right)$, the differential equation leads to

$$
\frac{\mathrm{d}^{2}}{\mathrm{~d} y^{2}} f_{j}(y)+\varepsilon f_{j}(y)=0
$$

It is possible to obtain a solution in terms of the regular perturbation according to

$$
f_{j}(y)=f_{j}^{0}(y)+\epsilon f_{j}^{1}(y)+\epsilon^{2} f_{j}^{2}(y)+\epsilon^{3} f_{j}^{3}(y)+\cdots
$$

The boundary conditions are imposed at the zero-th order. For higher-order terms, zero boundary conditions can be imposed.

$$
f_{j}^{0}(y)=\left(f_{j}(0)-f_{j}(1)\right) y+f_{0}(0) .
$$

Additional terms can be added as needed. This completes the solution for the first problem where only two of the boundary conditions are accounted for. The second problem can also be treated in a similar way.

${ }^{1}$ The case where $\sin (\sigma)=0$ is the degenerate case [13]. We are not treating that case here. 


\section{Numerical Experiments}

In this section, we use a numerical example to investigate the applicability of the proposed method. The proposed method is particularly useful for very high values of the wave number. For these values none of the existing numerical methods can be applied within the available computer capacity. It is possible to use a problem for which analytical solutions exist [2]. An exact solution for the problem is given by

$$
u(x, y)=\cos (k(x \cos (\theta)+y \sin (\theta)))
$$

where, $\theta$ is the angle of the incoming wave. Using the exact solution one can provide the boundary conditions for the numerical method. The domain is divided into equal intervals in both $x$ and $y$. Figure 2 presents the numerical results for $k=2000$. The figure shows the error as a function of the number of orthogonal functions. There is little dependency to the mesh size $n_{e}$. The error is the $L^{2}$ norm of the difference between the numerical result and the exact solution divide by the number of nodes.

Figures 3-5 show the same error reduction for higher wave numbers. Figure 3 presents the reduction in the error for $k=15000$. The number of intervals is equal to $n_{e}=1600$. For higher wave numbers, the same reduction in error is observed. For higher values of $k$, the method

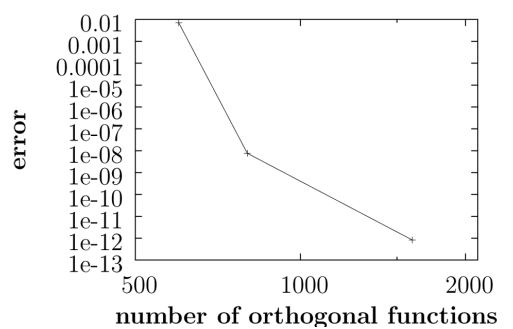

Figure 2. Reduction in the error as a function of the number of orthogonal functions. Other parameters are $k=2000, \theta=\pi / 4, n_{e}=800$, and the three maximum number of orthogonal functions are $n_{\text {orth }}=600,800,1600$.

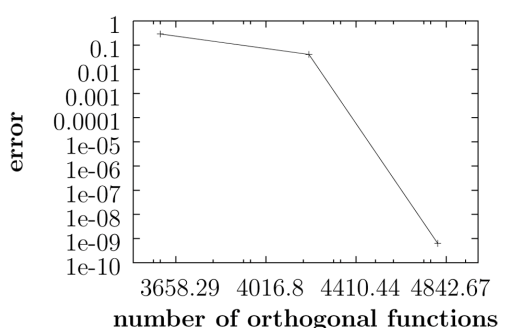

Figure 3. Reduction in the error as a function of the number of orthogonal functions. Other parameters are $k=15000, \theta=\pi / 4, n_{e}=1600$, and the three maximum number of orthogonal functions are $n_{\text {orth }}=3600,4200,4800$.

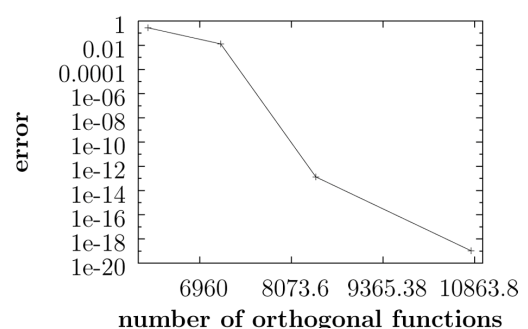

Figure 4. Reduction in the error as a function of the number of orthogonal functions. Other parameters are $k=25000, \theta=\pi / 4, n_{e}=1600$, and the three maximum number of orthogonal functions are $n_{\text {orth }}=6400,7200,8400,10800$.

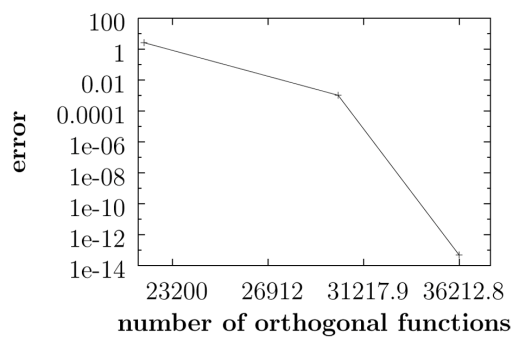

Figure 5. Reduction in the error as a function of the number of orthogonal functions. Other parameters are $k=100000, \theta=\pi / 4, n_{e}=1600$, and the three maximum number of orthogonal functions are $n_{\text {orth }}=22200,30000,8400,36200$.

simply requires the inclusion of more orthogonal functions in the expansion given in Equation (2). Figure 5 presents the result for $k=10^{5}$ and a similar reduction in the error is obtained.

The present method is particularly useful for the Helmholtz equation at higher frequency for which existing methods require a large amount of memory. The actual calculation for the present method is done analytically in one-dimension.

\section{Conclusion}

In this note, we presented a numerical method for the solution of 2-D Helmholtz equation. The method can be applied to 3-D domains. It is based on orthogonal functions. Apart from the projection of the problem onto the space of orthogonal functions, the solution is obtained analytically. Numerical results for a number of cases with very high wave numbers were presented.

\section{REFERENCES}

[1] G. E. Owen, "Introduction to Electromagnetic Theory," Dover, New York, 2003.

[2] I. Singer and E. Turkel, "High-Order Finite Difference Methods for the Helmholtz Equation," Computer Meth- 
ods in Applied Mechanics and Engineering, Vol. 163, No. 1-4, 1998, pp. 343-358.

http://dx.doi.org/10.1016/S0045-7825(98)00023-1

[3] M. Navabi, M. H. K. Siddiqui and J. Dargahi, "A New 9-Point Sixth-Order Accurate Compact Finite-Difference Method for the Helmholtz Equation," Journal of Sound and Vibration, Vol. 307, No. 3-5, 2007, pp. 972-982. http://dx.doi.org/10.1016/j.jsv.2007.06.070

[4] P. Nadukandi, E. Onate and J. Garcia, "A Fouth-Order Compact Scheme for the Helmholtz Equation: Alpha-Interpolation and FEM and FDM Stencils," International Journal for Numerical Methods in Engineering, Vol. 86, No. 1, 2011, pp. 18-46.

http://dx.doi.org/10.1002/nme.3043

[5] H. Dogan, V. Popov and E. Hin Ooi, "The Radial Basis Integral Equation Method for Solving the Helmholtz Equation," Engineering Analysis with Boundary Elements, Vol. 36, No. 6, 2012, pp. 934-943. http://dx.doi.org/10.1016/j.enganabound.2011.12.003

[6] X. Feng and H. Wu, "hp-Discontinuous Galerkin Methods for the Helmholtz Equation with Large Wave Number," Mathematics of Computation, Vol. 80, No. 276, 2011, pp. 1997-2024. http://dx.doi.org/10.1090/S0025-5718-2011-02475-0

[7] G. F. Dargush and M. M. Grigoiev, "A Multi-Level Multi-Integral Algorithm for the Helmholtz Equation," Proceedings of IMECE-2005, 5-11 November 2005, Orlando, pp. 1-8.

[8] K. Otto and E. Larsson, "Iterative Solution of the Helm- holtz Equation by a Second-Order Method," SIAM Journal on Matrix Analysis and Applications, Vol. 21, No. 1, 1999, pp. 209-229. http://dx.doi.org/10.1137/S0895479897316588

[9] D. Gordon and R. Gordon, "Robust and Highly Scalable Parallel Solution of the Helmholtz Equation with Large Wave Numbers," Journal of Computational and Applied Mathematics, Vol. 237, No. 1, 2013, pp. 182-196. http://dx.doi.org/10.1016/j.cam.2012.07.024

[10] Y. Zhuang and X. H. Sun, "A High-Order ADI Method for Separable Generalized Helmholtz Equations," Advances in Engineering Software, Vol. 31, No. 8-9, 2000, pp. 585-591.

http://dx.doi.org/10.1016/S0965-9978(00)00026-0

[11] B. N. Li, L. Cheng, A. J. Deek and M. Zhao, "A SemiAnalytical Solution Method for Two-Dimensional Helmholtz Equation," Applied Oscean Research, Vol. 28, No. 3, 2006, pp. 193-207. http://dx.doi.org/10.1016/j.apor.2006.06.003

[12] G. Bao, G. W. Wei and S. Zhao, "Numerical Solution of the Helmholtz Equation with High Wavenumbers," International Journal for Numerical Methods in Engineering, Vol. 59, No. 3, 2004, pp. 389-408. http://dx.doi.org/10.1002/nme.883

[13] Z. C. Li, "The Trefftz Method for the Helmholtz Equation with Degeneracy," Applied Numerical Mathematics, Vol. 58, No. 2, 2008, pp. 131-159. http://dx.doi.org/10.1016/j.apnum.2006.11.004

\section{Appendix}

In 3-D one can assume a form given by

$$
u(x, y, z)=\sum_{i=1}^{\infty} \sum_{j=1}^{\infty} f_{i j}(y) \sin (i \pi x) \sin (j \pi z),
$$

here, after using the orthogonality conditions, the func-

tions $f_{i j}(y)$ are given by

$$
f_{i j}(y)=4 \int_{0}^{1} \int_{0}^{1} u(x, y, z) \sin (i \pi x) \sin (j \pi z) d x d z
$$

Also, for the 3-D Helmholtz equation, one needs to split the problem into three similar problems. 